\title{
The Usage of Banana (Musa Acuminata) for Novel Beverage Production
}

\author{
Nkerbu Rodolphe Ngwang* \\ Department of Food Biotechnology, Assumption University, Thailand \\ * Corresponding author. Tel.: (662)719-199; email: tankou83@yahoo.fr. \\ Manuscript submitted October 11, 2014; accepted December 18, 2014. \\ doi: 10.17706/ijbbb.2015.5.1.62-69
}

\begin{abstract}
The quality properties of alcoholic beverages made from ripe banana were examined. Long and short hand banana (LHB and SHB) of the genus musa were used. Juice was extracted from the banana pulps using distilled and mineral water respectively. The extracted juices contained $15^{\circ} \mathrm{brix}, \mathrm{pH}$ ranged between 4.28 and 4.58 , titratable acidity (TA) ranged between 0.23 and $0.27 \mathrm{~g} / 100 \mathrm{ml}$. LHB extract was light yellow while SHB extract was dark yellow in color. LHB extracts aromas were fruitier and sweeter than SHB extracts. Part of each extract was fermented at $(30 \pm 2)^{\circ} \mathrm{C}$ by adding wine yeast (Saccharomyces cerevisiae) and another, a combination of both wine yeast and lactic acid bacteria (LAB). It took 7 to 10 days for complete fermentation. Soluble solids (SS) and pH decreased while (TA) increased with Time. The banana beverage produced had values that ranged from 3.45 to 4.04 for $\mathrm{pH}, 0.77$ to $1.1 \mathrm{~g} / 100 \mathrm{ml}$ for TA, $6{ }^{0} \mathrm{Brix}, 5.5$ to 6.45 for alcohol (v/v) percentage (\%). Results revealed no significant difference in $\mathrm{pH}, \mathrm{TA}$, and alcohol content of the beverages produced $(p>0.05)$, there is a significant difference between mineral water LHB juice extract fermented by adding both yeast and LAB and the distilled water fermented extracts $p<0.05$. LHB juice extracted fermented by adding both yeast and LAB was the most preferred product. An alcoholic beverage was successfully produced from banana.
\end{abstract}

Key words: Banana, fermentation, lactic acid bacteria, Saccharomyces cerevisiae.

\section{Introduction}

Banana belongs to the family Musaceae and genus Musa. Musa spp., which has already provided man with food, tools and shelter prior to recorded history. Banana is a major crop of West and East Africa and is grown in some 120 countries throughout the developing world. It is a fruit common in the tropics and is non-seasonal [1]. There are several types of bananas grown for food consumption. These have been classified as cooking bananas, roasting bananas, sweet bananas and brewing bananas [2]. According to the latest FAO statistical records as reported by the International Institute of Tropical Agriculture, more than 58 million tons of bananas and 30 million tons of plantains were produced worldwide in 2000 [3]. India is the largest banana producer with an output of 16 million tons per annum and Uganda ranks second for producing 12 million tons per annum [3] [4]. Depending upon cultivar and ripeness, the flesh can vary in taste from starchy to sweet and texture from firm to mushy. Both skin and inner part can be eaten raw or cooked. Though banana is mainly known for its fruit as a culinary ingredient, it is classified as a functional food because it contains a food component that affects one or a limited number of function(s) in the body in a targeted way so as to have positive effects [5]. Banana possesses desirable qualities such as high fiber- 
content which helps restore normal bowl action, stimulates the production of hemoglobin in the blood. It contains potassium and has a low salt content which helps to lower blood pressure as well as control stroke and when consumed along with other fruits and vegetables and banana was observed to be associated with reduced risk of various cancers such as renal cell carcinoma [6]. There is considerable waste of the banana especially those that do not meet the quality norms for export. This is due to the fact that the banana fruit is biologically active and carries out transpiration, ripening and other biochemical activities even after harvests [7]. This phenomenon is turned to deteriorate the quality of the fruit and finally makes it unmarketable. Wastage of the banana fruit due to poor post-harvest handling or over-ripening remains a major problem today. The conversion on banana into other valuable products before spoilage has been the driving force behind this study knowing enough bananas is wasted after post harvesting. In the course of this study we decided to focus on the production of an alcoholic beverage from two varieties of ripe banana using microorganisms like yeast and Lactic acid bacteria and two types of extraction solvents( distilled and mineral water). The objective here was to find out if there exists a difference between the quality of the short and long hand banana, the types of liquid used and the types of microorganisms used in the process.

\section{Materials and Methods}

\subsection{Banana Cultivars Used and Juice Preparation}

Two banana types of the genus musa at the same stage of ripeness were purchased in a local market in Thailand and used, which are long hand and short hand banana respectively. Bananas were peeled off and masses $2000 \mathrm{~g}$ of the pulps were weighed. The pulps were then sliced into pieces and $50 \%$ distilled water was added to each banana pulp weighed. The mixtures were ground in a blender and $0.005 \mathrm{ml}$ of pectinase (16000 PECTU/ml) was added. Ref. [8] reported that the addition of pectinase enzymes in the preparation of wines served to reduce viscosity, facilitated pressing of fermented solids and in so doing increased the yield. The containers were covered and allowed to stand overnight at room temperature. After addition of enzyme the mixtures were allowed to stand at ambient temperatures for 24 hours. The mixtures were then filtered using a muslin-cheese cloth and the juice extracted. Prior to inoculation and fermentation, physiochemical properties of the extract were measured.

\subsection{Preparation of the Yeast Starter Culture}

Yeast was grown in yeast mold agar. We prepared a $0.1 \%$ peptone solution. To $20 \mathrm{ml}$ of this peptone solution we added $0.5 \mathrm{~g}$ of dry wine yeast. We prepared further decimal dilution to $10^{-3}$ and allowed to stand overnight. The next day we agitated the tube containing the mixture by means of a vortex and we plated $0.1 \mathrm{ml}$ on the YM agar which had previously been autoclaved and put in plates and allowed to solidify. The plates were then incubated at $37{ }^{\circ} \mathrm{C}$ for 48 hours. After determining the wine yeast cell growth in the medium, the cells were re-suspended in $10 \mathrm{ml}$ of distilled water. The banana pulp was inoculated at $2 \times 10^{6}$ cells per ml.

\subsection{Isolation and Growth of Lactic Acid Bacteria from Yoghurt}

We purchased natural yoghurt from Food land (Yalida) containing probiotics i.e. life bacteria cultures of Lactobacillus bulgaricus and Staphylococcus thermophiles. We suspended 1g of yoghurt in MRS broth and it was incubated at $37^{\circ} \mathrm{C}$ for $24 \mathrm{~h}$. We prepared further decimal dilution to $10^{-3}$. The next day we plated $0.1 \mathrm{ml}$ of the content of the broth on MRS agar plates which had previously been prepared and allowed to solidify and we incubated the plates again at $37^{\circ} \mathrm{C}$ for 48 hours until growth appeared. After determining the lactic acid bacteria cell growth in the medium, the cells were re-suspended in $10 \mathrm{ml}$ of distilled water. The banana pulp was inoculated at $1.6 \times 10^{6}$ cells per ml. 


\subsection{Pitching and Fermentation of the Juice Extracted}

From each banana extract, long and short hand respectively, we prepared 2 parts of volume $350 \mathrm{ml}$. To one part we added $0.5 \mathrm{ml}$ of yeast, to the other we added $0.5 \mathrm{ml}$ yeast and LAB respectively. The various formulations are given in Table 1 below. We monitored the evolution of various parameters during the fermentation. Fermentation end point was determined when the ${ }^{0}$ Brix remained constant. It should be noted that this was done in duplicate. After complete fermentation, the juice was clarified by filtration using vacuum aspirator. We used a vacuum pump (Its types/s $\left[\mathrm{m}^{3} / \mathrm{h}\right] \mathrm{ME} 2 \mathrm{c} / 1,7$, ser no 19028206/95) and what man filter paper 4 of pore diameter $110 \mathrm{mn} \phi$ to get rid the suspended particles and consequently obtain a clearer solution. After comparison of LHB and SHB fermented extracts, the best banana extract (LHB) was taken for further study and analysis. As previously mentioned above, LHB pulp juice was extracted using distilled and mineral water respectively. Using the same amount of juice as above, fermentation was done as previously mentioned by inoculating part of each extract with yeast and another with both yeast and LAB respectively.

Table 1. LHB Fermented Extracts Formulations

\begin{tabular}{lllll}
\hline \hline Banana type & sample & Solvent & Microorganism & Fermented volume \\
\hline LHB & 1 & Mineral water & Yeast & $350 \mathrm{ml}$ \\
& 2 & Mineral water & Yeast/LAB & $350 \mathrm{ml}$ \\
LHB & 1 & Distilled water & Yeast & $350 \mathrm{ml}$ \\
& 2 & Distilled water & Yeast/LAB & $350 \mathrm{ml}$ \\
\hline \hline
\end{tabular}

\subsection{Physicochemical Analyses of Banana Wine}

The $\mathrm{pH}$ of the wine was measured by using a glass calomel electrode $\mathrm{pH}$ meter, titratable acidity was measured by AOAC Physico-chemical analysis method [9]. The soluble solids ( ${ }^{\circ} \mathrm{brix}$ ) in the wine were determined using a hydrometer [10] and the alcohol content was measured in volume percentage (\%v/v) by ebulliometry [11].

\subsection{Preference Test}

Panel test was conducted in the sensory evaluation laboratory of Assumption university. 30 panelists for each sample were asked to participate in the study. A 4-point hedonic scale questionnaire was used to score the liking of each sample. All the samples were served and placed in front of each panelist at the same time. Alongside we placed a cup of mineral water which we advised the panelist to rinse their mouth with before starting the test as well as in between two different samples to be tested. A form containing the instructions to follow was given to each subject. We asked the panelists to begin with the sample on their left and also that they could restart the tasting as often and they needed to. After they had finished tasting, they could start ranking the samples from most preferred (4) to least preferred (1).

\subsection{Statistical Analysis}

Analysis of preference test data was carried on using SPSS trial version.

\section{Results and Discussion}

Results of the comparison between LHB and SHB fermented extracts are given in Table 2 below. It shows us that there was no significant difference in $\mathrm{pH}, \mathrm{TA},{ }^{0} \mathrm{Brix}$ and percentage alcohol $(\% \mathrm{v} / \mathrm{v})$ of various fermented samples ( $p>0.05)$, and there was significant difference in color $p<0.05$ for the long 
hand banana extract (light yellow) and short hand banana extracts (dark yellow). There was a significant difference in aroma of LHB and SHB $(p<0.05)$. The aroma of LHB was dominant over SHB for reasons we still need to explore even though we can think of the conditions of cultivation and the banana variety as part of the cause.

Table 2. Average Values of Parameters of LHB and SHB Fermented Extracts (Mean \pm SD)

\begin{tabular}{ccccc}
\hline parameters & SHB/yeast & SHB/yeast/LAB & LHB/yeast & LHB/yeast/LAB \\
\hline pH & $3.94 \pm 0.057^{\mathrm{a}}$ & $3.85 \pm 0.028^{\mathrm{a}}$ & $4.02 \pm 0.042^{\mathrm{a}}$ & $4.04 \pm 0.014^{\mathrm{a}}$ \\
TA g/100ml & $0.94 \pm 0.014^{\mathrm{a}}$ & $0.95 \pm 0.014^{\mathrm{a}}$ & $0.8 \pm 0.028^{\mathrm{a}}$ & $0.77 \pm 0.113^{\mathrm{a}}$ \\
0BRIX & 6 & 6 & 6 & 6 \\
ALCOHOL & $6.45 \pm 0.071^{\mathrm{a}}$ & $6 . \pm 0.282^{\mathrm{a}}$ & $6.3 \pm 0.707^{\mathrm{a}}$ & $5.5 \pm 0.424^{\mathrm{a}}$ \\
& $3.35 \pm 0.0291^{\mathrm{b}}$ Dark & $3.38 \pm 0.0172^{\mathrm{b}}$ Dark & $4.11 \pm 0.043^{\mathrm{b}}$ Light $^{\mathrm{a}}$ & $4.41 \pm 0.4$ Light yellow \\
Color & yellow & yellow & yellow & \\
& $3.20 \pm 0.273^{\mathrm{b}}$ & $3.20 \pm 0.273^{\mathrm{b}}$ & $4.10 \pm 0.512^{\mathrm{a}}$ & $4.31 \pm 0.04^{4 a}$
\end{tabular}

SHB: Short hand banana LHB: Long hand banana LAB: lactic acid bacteria

The changes in $\mathrm{pH}$ of the LHB fermented extracts used for further studies and analysis as given in Fig. 1 and Fig. 2 below.

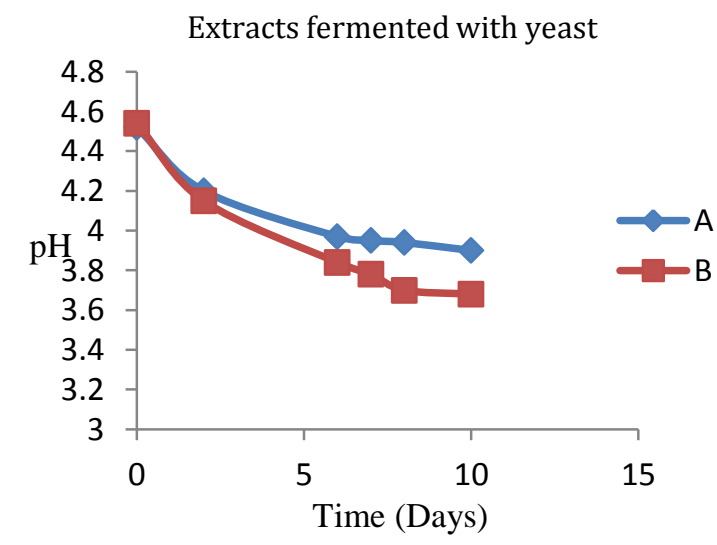

Fig. 1. The change of $\mathrm{pH}$ in LHB extract fermented by adding with yeast, $A=$ LHB/distilled water inoculated with yeast, $B=$ LHB/ mineral water inoculated with yeast.

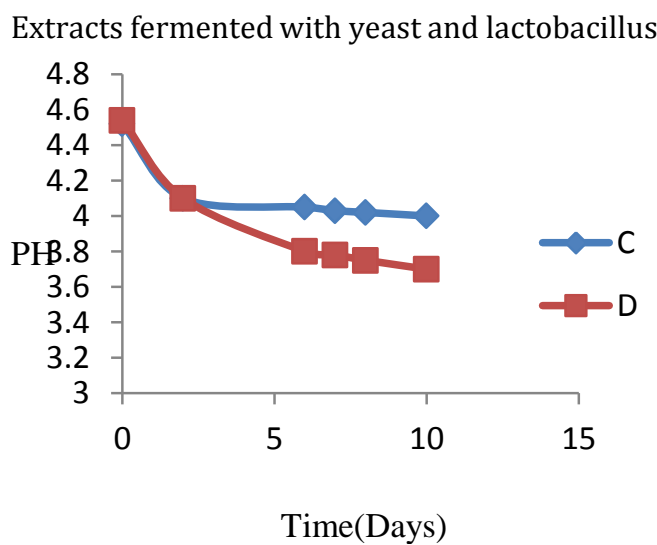

Fig. 2. The change of $\mathrm{pH}$ of banana extract fermented by adding yeast and LAB. C= LHB/distilled water extract inoculated with both yeast and LAB, D= LHB/ mineral water extract inoculated with both yeast and LAB.

It was observed that the $\mathrm{pH}$ values decreased with fermentation time apparently due to the production of acids from the fermentation. This result agrees with the reports of Ref. [3]. The initial pH was 4.52 for the LHB/distilled water extract and 4.54 for banana/mineral water extract. At the end of the fermentation, the samples containing mineral water scored lower $\mathrm{pH}$ than those fermented with distilled water even though overall there was no significant difference in $\mathrm{pH}$ of the various products $(p>0.05)$. Inverly, It was observed in Fig. 3 and Fig. 4 below that titratable acidity increased with period of fermentation. It was also observed that the samples fermented with both yeast and lactic acid bacteria had acidities higher than those fermented with yeast only. This could be attributed to the presence of a lactic acid fermentation arising from succession of the yeast cells by lactic acid bacteria during fermentation. 


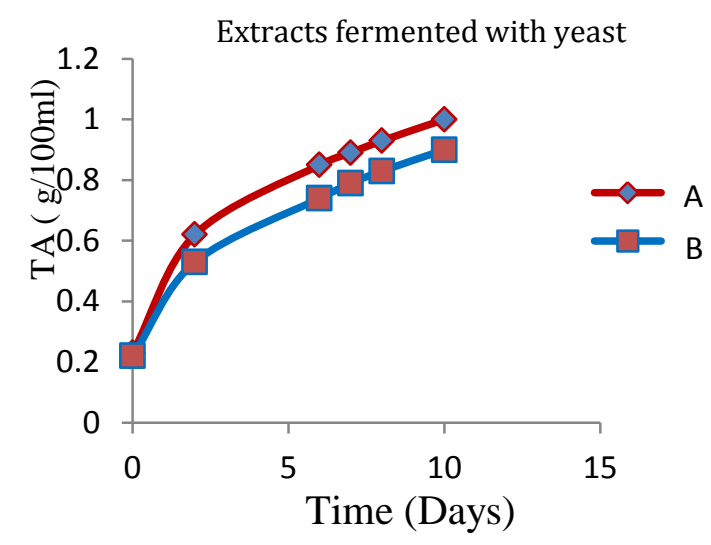

Fig. 3. The change of TA with time of extract fermented by adding yeast, A=LHB/distilled water inoculated with yeast, $\mathrm{B}=\mathrm{LHB} /$ mineral water inoculated with yeast.
Extracts fermented with yeast and lactobacillus

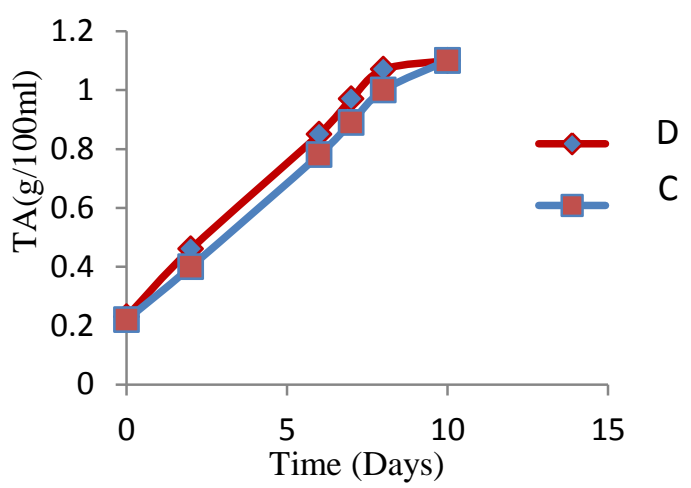

Fig. 4. The change of TA with time of extract fermented by adding yeast and LAB, C= LHB/distilled water extract inoculated with both yeast and LAB, D= LHB/ mineral water extract inoculated with both yeast and LAB.

Inoculating musts with yeast and lactic acid bacteria (LAB) concurrently in order to induce simultaneous alcoholic fermentation (AF) and lactic acid fermentation (MLF) can be an efficient alternative to overcome potential inhibition of LAB in wines because of high ethanol concentrations and reduced nutrient content [12]. There was no significant difference between the acidity of the various samples $(p>0.05)$.

Extracts fermented with yeast

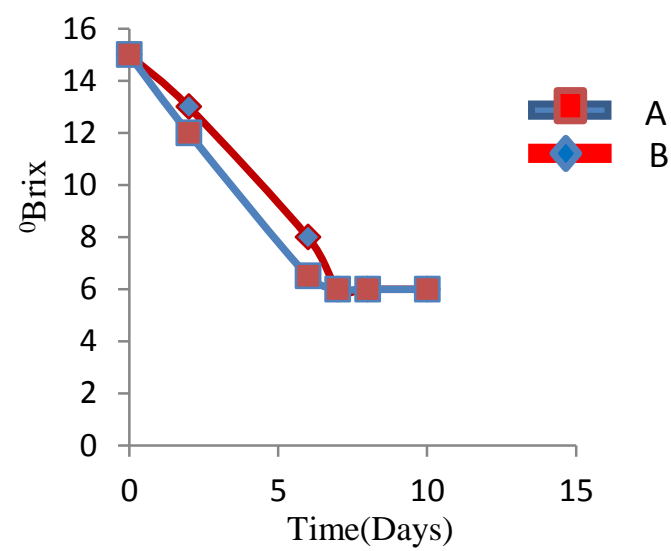

Fig. 5. The change of ${ }^{0}$ Brix in extracts inoculated with yeast, $A=\mathrm{LHB} /$ distilled water inoculated with yeast, $\mathrm{B}=\mathrm{LHB} /$ mineral water inoculated with yeast.
Extracts fermented with yeast and lab

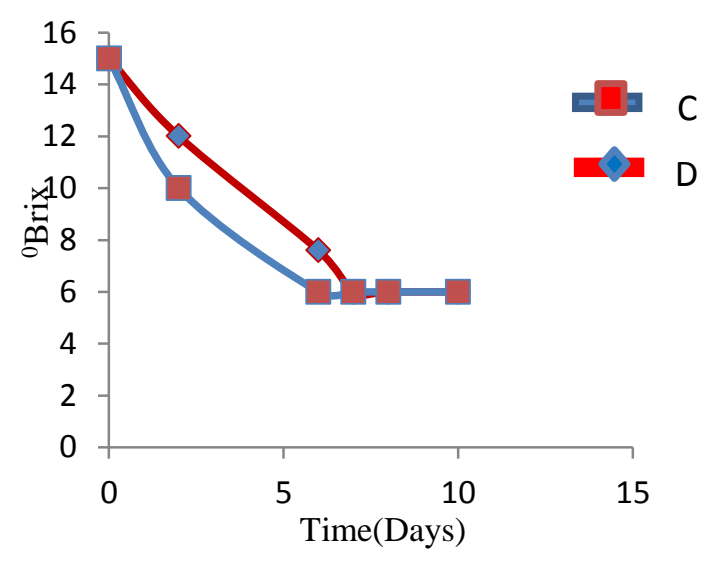

Fig. 6. The change of ${ }^{0}$ Brix in extract inoculated with yeast and $\mathrm{LAB} \mathrm{C}=\mathrm{LHB} /$ distilled water extract inoculated with both yeast and LAB $\mathrm{D}=\mathrm{LHB} /$ mineral water extract inoculated with both yeast and LAB.

Fig. 5 and Fig. 6 above show us that ${ }^{0}$ Brix decreases with time and the rate at which the ${ }^{0}$ Brix decreasd with time was greater for the sample $C$ and D than for sample A and B. This may be due to both starter culture competing for nutrients thus rapid consumption of sugar molecules such as glucose unlike in the samples A and B which only contained yeast as the starter culture. There was also a slight reduction in fermentation time observed in A and C compared to sample B and D. i.e. it took about 7 to 10 days to reach a constant sugar level unlike 8 to 10 days in the samples B and D. This could be accounted for the type of 
water (mineral water) that most have provided some kind of optimum condition for the cultures to grow and consume the sugar molecules [13]. Minerals increase the yeast's ability to metabolize or consume sugar. Without a supply of minerals you have yeasts that consume sugar at a slower pace. Using distilled water can cause big problems for the unsuspecting winemaker. There are two reasons for this. The first being distilled water has had all of the excess or free oxygen removed from it. The second reason is that distilled water has no minerals either. Both of these conditions are direct results of the distilling process and both conditions have inhibiting effects on fermentation [13].

The alcohol percentages $\%(\mathrm{v} / \mathrm{v})$ of the banana beverage after fermentation is shown in Table 3 below. It was observed that the sample inoculated with both yeast and LAB scored a little less alcohol content compared to those inoculated with only yeast (A 6.4\%, B 5.9\%, C 6.3\%, D 5.8\%) the slightly low alcohol content might be due to the combine effect of both yeast and LAB. lactic acid fermentation can occur spontaneously as a result of LAB that occurs naturally, or it can be induced with commercial starter cultures. Yeasts produce compounds i.e. ethanol, $\mathrm{SO}_{2}$ and medium chain fatty acids that inhibit the growth of LAB. Both yeasts and LAB compete with each other for nutrients, thus leading to low alcohol content in samples inoculated with both starter cultures, since the LAB consumes some of the sugar to produce lactic acid which of course would have been used by yeast to produce ethanol thus leading to a lower alcohol content. This agrees with Ref. [14]. But from an overall perspective, there was no significant difference between the alcohol contents of the various samples $(p \geq 0.05)$. There was also a decrease in aroma of the various wines (sweetness), we could simply attribute this to the volatility of the sweet banana aromatic compounds. The aroma responsible for the flavor of fruits is highly susceptible to the temperatures in storage [15].

Table 3. Average Values of Parameters of Improved LHB Beverages (Mean \pm SD)

\begin{tabular}{ccccc}
\hline parameters & A & B & C & D \\
\hline pH & $3.45 \pm 0.212^{\mathrm{a}}$ & $3.68 \pm 0.113^{\mathrm{a}}$ & $3.7 \pm 0.283^{\mathrm{a}}$ & $4 \pm 0.282^{\mathrm{a}}$ \\
TA g/100ml & $1 \pm 0.141^{\mathrm{b}}$ & $0.9 \pm 0.141^{\mathrm{b}}$ & $1.1 \pm 0.354^{\mathrm{b}}$ & $1.1 \pm 0.212^{\mathrm{b}}$ \\
0BRIX & 6 & 6 & 6 & 6 \\
ALCOHOL\% & $6.4 \pm 0.566^{\mathrm{a}}$ & $5.9 \pm 0.141^{\mathrm{a}}$ & $6.3 \pm 0.283^{\mathrm{a}}$ & $5.8 \pm 0.141^{\mathrm{a}}$ \\
\hline
\end{tabular}

Table 4. Mean of Overall Liking of Each Sample

\begin{tabular}{ll}
\hline \hline samples & Mean \pm S.D \\
\hline A & $2.33 \pm 1.09334^{\mathrm{a}}$ \\
B & $2.7 \pm 1.02217^{\mathrm{ab}}$ \\
C & $2.066 \pm 1.11211^{\mathrm{a}}$ \\
D & $2.9 \pm 1.12495^{\mathrm{b}}$ \\
\hline \hline
\end{tabular}

$\mathrm{A}=\mathrm{LHB} /$ distilled water extract fermented by adding yeast, $\mathrm{B}=\mathrm{LHB} /$ mineral water extract fermented by adding yeast, $\mathrm{C}=$ $\mathrm{LHB} /$ mineral water extract fermented by adding both yeast and LAB, D= LHB/ distilled water extract fermented by adding both yeast and LAB.

As shown in Table 4 above, Sample D was the most liked while sample $C$ was the least liked. Results also revealed that there was no significant difference between $A, B$ and $C(p \geq 0.05)$, meanwhile D was significantly different to $A$ and $C(p \leq 0.05)$, but none significantly different to $B(p \geq 0.05)$.

\section{Conclusion}


Banana was successfully used to produce an alcoholic beverage. The beverages showed no appreciable difference in $\mathrm{PH}, \mathrm{TA}$, percentage (\%) alcohol (v/v), obrix p.0.05. LHB extract color and aroma were significantly different to LHB extract $(p<0.05)$. Mineral water fermented extracts were preferred to distilled water fermented extract, also the combination of mineral water and both wine yeast and LAB resulted in the most liked beverage. This study could be extended using chromatography to identifying the compounds present in the beverage after fermentation and eventually their health benefits, this might also help us to explain why LHB extracts are more aromatic than SHB extracts. There is also the need for establishing shelf life of the products.

\section{References}

[1] Byarugaba-Bazirake, W. G., Byarugaba, W., Tumusiime, M., \& Kimono, D. A. (2014). The technology of producing banana wine vinegar from starch of banana peels. African Journal of Food Science and Technology, 5(1), 1-5.

[2] Emmanuel, O. I., \& Ikenna, E. O. (2011). Studies of wine produced from banana. International Journal of Biotechnology and Molecular Biology, 2(12), 209-214.

[3] Byarugaba-Bazirake, W. G. (2008). The Effect of Enzymatic Processing on Banana Juice and Win. Dissertation presented for the degree of doctor of philosophy, Stellenbosch University.

[4] FAO, world banana economy, 1985-2002, retrieved august 20, 2014, from the website: http://www.fao.org/3/a-y5102e/y5102e04.htm\#TopOfPage.

[5] Mohammad, S. Y., Salmah, Y., Mohd, A. B. Y., \& Abd-aziz, S. (2009). Storage stability of clarified banana juice fortified with inulin and oligofructose. Journal of Food Processing and Preservation, 34, 599-610.

[6] Rashidkhani, B., Åkesson, A., Lindblad, P., \& Wolk, A. (2005). Fruits, vegetables and risk of renal cell carcinoma a prospective study of Swedish women. Int. J. Cancer, 113(3), 451-455.

[7] Hailu, M., Workneh, T. S., \& Belew, D. (2013). Review on postharvest technology of banana fruit. African Journal of Biotechnology, 12(7), 635-647.

[8] Tapre, A. R., \& Jain, R. K. (2012). Optimization of process variables for enzymatic clarification of banana pulp. International Journal of Emerging trend in Engineering and Development, 2 (7), 319-330.

[9] A.O.A.C., 1990, Official Methods of Analysis (15th ed.) Arlington: Association of official Analytical Chemists, Inc.

[10] Iland, P., Ewart, A., Sitters, J., Markides, A., \& Bruer, N. (2000). Techniques for Chemical Analysis and Quality Monitoring During Winemaking. Australia: Patrick Iland Wine Promotions.

[11] Kane, A. (2013). Winemaking 301 - Advanced. Presque Isle Wine Cellars, p: 1- 31.

[12] Jussier, D., De Orduña, R. M., \& Morneau, A. D. (2006). Effect of simultaneous inoculation with yeast and bacteria on fermentation kinetics and key wine parameters of cool climate chardonnay. Applied Environmental Microbiology, 72(1), 221-227.

[13] Kraus, E., \& Cblog. (2013). Top 10 reasons for fermentation failure, home wine and beer making supplies. Retrieve on may 20,2013, from the website: http://www.eckraus.com/wine-making-stuck-9.

[14] Heinrich, P. H., \& Neil, J. (2000). Influence of wine yeast NT202 on the progress of malolactic fermentation. A technical guide for wine fermentation, wine land publication.

[15] Facundo Vasconcelo, H. V. D., Garruti, D. D. S., Carlos Tadeu, D. S. D., Beatriz R. C., \& Franco, M. L. (2012). Influence of different banana cultivars on volatile compounds during ripening in cold storage. Food Research International Journal, 49, 626-633. 
Nkerbu Rodolphe Ngwang is a master degree student at the Faculty of Biotechnology, Department of Food Biotechnology, Assumption University of Thailand. He was originally from Cameroon where he obtained a bachelor of science in biochemistry at the University of Douala, Cameroon in 2006. Presently he is working on the publication of his research paper to officially graduate with a master of science in food biotechnology. 